Document downloaded from:

http://hdl.handle.net/10251/82264

This paper must be cited as:

Guardiola, C.; Dolz García, VM.; Pla Moreno, B.; Mora-Pérez, J. (2016). Fast estimation of diesel oxidation catalysts inlet gas temperature. Control Engineering Practice. 56:148-156. doi:10.1016/j.conengprac.2016.08.020.

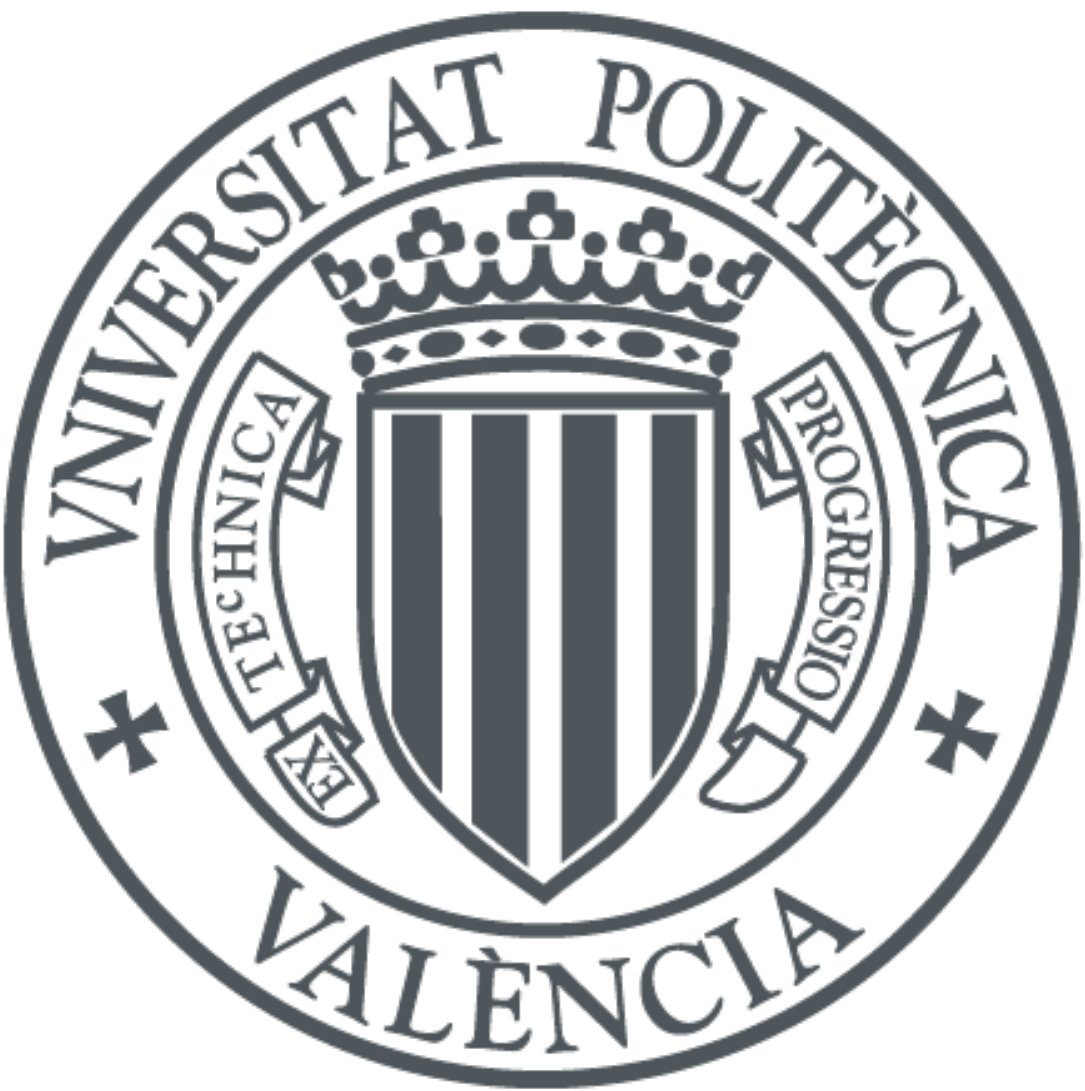

The final publication is available at

http://doi.org/10.1016/j.conengprac.2016.08.020

Copyright Elsevier

Additional Information 


\title{
Fast estimation of diesel oxidation catalysts inlet gas temperature
}

\author{
C. Guardiola ${ }^{a}$ V. Dolz ${ }^{\text {a }}$ B. Pla ${ }^{\text {a J. Mora }}{ }^{a}$ \\ ${ }^{a}$ CMT-Motores Térmicos, Universitat Politècnica de València, Valencia, Spain (e-mail: \\ \{carguaga,vidolrui, benplamo,jamopre1\}@mot.upv.es).
}

\begin{abstract}
With the tightening of on-board diagnostics requirements, accuracy of sensors is essential to monitor the efficiency and ensure a proper control of the after-treatment systems. Temperature sensors are commonly used in the exhaust line at the diesel oxidation catalyst-inlet of turbocharged diesel engines for control and diagnosis of the after-treatment system. In particular, negative temperature constant sensors are used for this purpose. However, due to the necessary on-board robustness that sensors must fulfil, thermal inertia causes significant differences during engine transient operating conditions in temperature measurements. A Kalman filter is proposed in this paper for the on-line dynamic estimation of the catalyst-inlet temperature, which combines a slow but accurate measurement of the on-board temperature sensor with a fast but drifted temperature model. A fast research-grade thermocouple is used as reference of the actual exhaust gas temperature and a frequency analysis is performed in order to calibrate the model and analyse results of the signal reconstruction. Results of the algorithm are then successfully proved in experimental transient tests and typical European approval test cycles.
\end{abstract}

Key words: on-board, temperature, fast estimation, control, turbocharged engine, diesel oxidation catalyst

\section{Introduction}

Temperature plays a key role in the efficiency of aftertreatment systems: Diesel Oxidation Catalysts (DOC) have an activation threshold, referred as Light-Off Temperature (LOT), which has to be overcome to oxidise $\mathrm{HC}, \mathrm{CO}$ and NO species [1]; Selective Catalytic Reduction (SCR) has to operate in a temperature band to ensure high conversion efficiency [2]; and Diesel Particulate Filters (DPF) regeneration needs an accurate closed loop control of the temperature $[3,4]$. As DOCs efficiency affects the performance of the rest of after-treatment systems [5], two temperature sensors have been traditionally used for control and OnBoard Diagnostics (OBD) purposes $[6,7,8]$. One is placed at the DOC inlet and the other at the outlet.

Increasing emissions standards for Diesel engines $[9,10]$ makes manufacturers struggle to find an after-treatment system configuration which optimizes the trade-off between low cost, low emissions, low fuel consumption and robustness [11]. Temperature sensors must be robust enough to stand up to the vehicle's life [12]. These robustness requirements are satisfied by increasing the sensor mass, whose thermal inertia implies filtering the exhaust gas temperature measurements. Negative Temperature Constant (NTC) sensors are the traditional sensors used in produc-

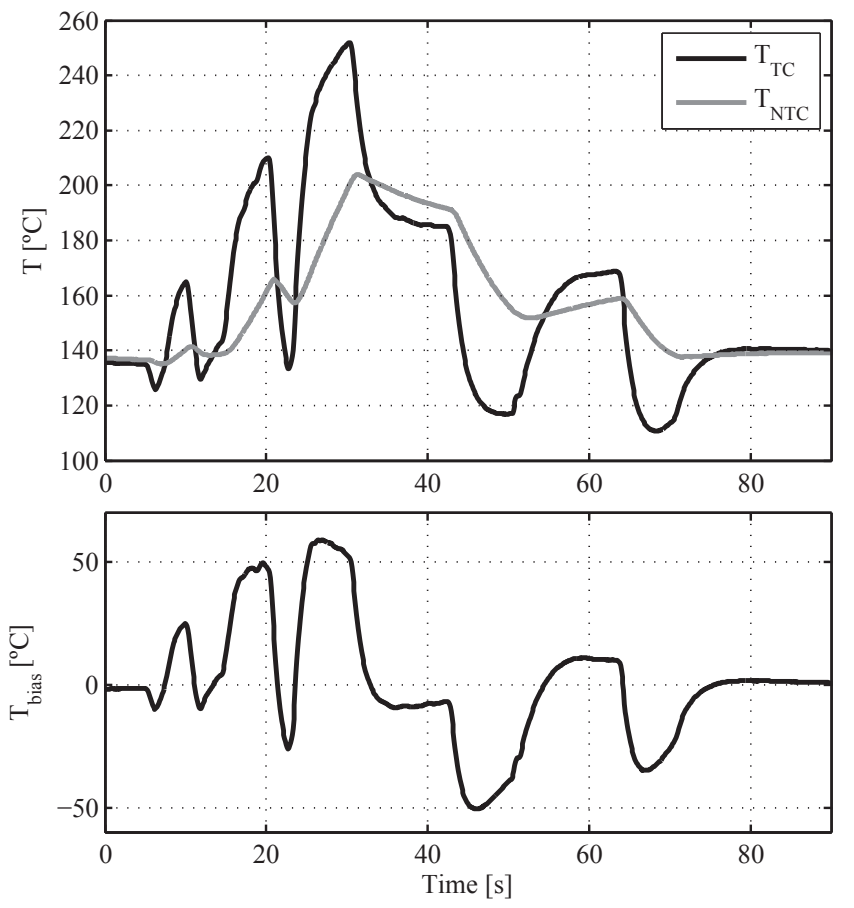

Fig. 1. Top plot: Temperature measurements during a time window of a NEDC. Bottom plot: $T_{T C}-T_{N T C}$ during the time window. 
tion vehicles for measuring exhaust gas temperature [13].

Figure 1 represents the DOC-inlet temperature evolution during a temporal window of the New European Driving Cycle (NEDC). $T_{T C}$ represents the signal provided by a thin thermocouple (TC), whilst $T_{N T C}$ represents the signal of the NTC sensor. It can be observed how the NTC sensor is not able to properly capture the temperature dynamics. Peak errors between the NTC sensor measurement and the thermocouple are over $\pm 50^{\circ} \mathrm{C}$, as shown in the bottom plot of Figure 1. If these differences occur near the LOT of the DOC, which is around $200^{\circ} \mathrm{C}$ [16], as it happens in Figure 1, a DOC model using the NTC measurement as input would not estimate any oxidation, which would lead to undesired errors in the DOC efficiency monitoring and diagnosis. Authors like Kar et al. [14] and Kee et al. [15] adressed solutions to improve temperature measurements during transient conditions by a reconstruction technique based on applying an energy balance to the heat transfer around two thermocouples at the same place. However, onboard limitations do not allow the use of two temperature sensors.

As it happens with temperature measurements, concentration sensors are not fast enough to measure exhaust gas composition in transient conditions. The contribution of the transient emission peaks during the standardized test cycles becomes in an important fraction of the cumulative total emissions [17]. Therefore, the problem of the dynamic response of concentration sensors has been addressed during last years. For instance, techniques like the combination of sensors and models in order to rebuild a signal are being commonly used in recent years in automotive industry [18]. Authors like Alberer [19] and Guardiola et al. [20] have researched diesel engine exhaust $\mathrm{O}_{2}$ and $\mathrm{NO}_{x}$ concentrations with special focus in transient conditions through the use of oxygen (UEGO) and $\mathrm{NO}_{x}$ sensors, which are widespread in the state of the art vehicles. These authors have determined a wide methodology of data fusion from sensors and models by using filtering techniques in order to have fast estimations of exhaust gas concentrations.

This work addresses the trade-off between robustness and dynamic response of on-board temperature sensors. In particular, the goal is to have a good transient response using a robust NTC sensor. To this aim, the NTC sensor provides a reliable but slow measurement, whilst a non-necessarily precise but fast model, based on real-time ECU engine variables, provides dynamic information. The NTC sensor is located upstream the after-treatment systems while the fast model is based on a look-up table depending on injected fuel quantity and engine speed.

While this paper is focused on the DOC for being the first after-treatment system placed in the exhaust line, this methodology could be also applicable to other exhaust line configurations with different after-treatment platforms. In fact, the fast temperature estimation provides a better measurement of the thermal energy entering in the system at the location of the NTC sensor.

The paper is structured as follows. Section 2 describes
Table 1

Engine characteristics.

\begin{tabular}{ll}
\hline Stroke (S) & $88 \mathrm{~mm}$ \\
Bore (D) & $85 \mathrm{~mm}$ \\
S/D & 1.036 \\
Number of cylinders (z) & 4 \\
Displacement & $1997 \mathrm{~cm}^{3}$ \\
Turbocharging system & $\mathrm{VGT}$ \\
Valves by cylinder & 4 \\
Maximum power & $120 \mathrm{~kW} @ 3750 \mathrm{rpm}$ \\
Compression ratio & $16: 1$ \\
\hline
\end{tabular}

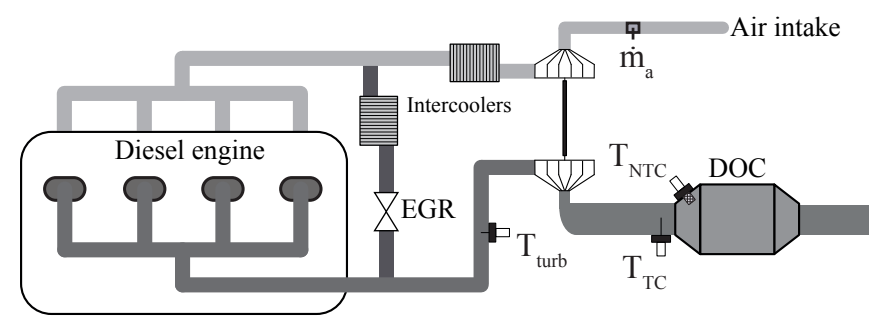

Fig. 2. Engine air path and on-purpose sensors layout at the test bench.

the experimental set-up. Section 3 explores engine dynamics in order to select suitable signals for the fast temperature model. Section 4 identifies the coefficients of the modelled systems. Section 5 contains the description of the used filtering technique and its calibration procedures. Section 6 contains results of the temperature estimation. Finally, section 7 contains the conclusions.

\section{Experimental set-up}

Experimental tests were carried out in a Euro IV direct injection diesel engine, installed on an engine test bench. Characteristics of the engine, mainly composed of a high pressure common-rail fuel injection system, an Exhaust Gas Recirculation (EGR) system and a Variable Geometry Turbine (VGT), are described in Table 1. The DOC was the only after-treatment system placed in the exhaust line. The pressure drop originated by a DPF and an SCR was generated by a back pressure valve. Experiments shown in this article consist of dynamic cycles and load steps that allow characterising the system dynamics.

As shown in Figure 2, the main sensors used in this work were a thin thermocouple, an NTC sensor placed at the DOC-inlet and other signals available from the ECU. The thermocouple is a $K$ type sensor with naked wires of $0.5 \mathrm{~mm}$ width and 3 meters long (model $T C 1 W C-K-3 \mathrm{~m}$ ), able to measure in a range of temperatures from $0^{\circ} \mathrm{C}$ to $1100^{\circ} \mathrm{C}$. The fast response was obtained at the cost of having to replace the sensor several times during experimentation due to its low robustness. Additionally, a thermocou- 
Table 2

Signals description

\begin{tabular}{ll}
$T_{\text {turb }}$ & Temperature measured by a thermocouple at \\
& the turbine inlet. \\
$T_{T C}$ & Temperature measured by a thermocouple at \\
& the DOC inlet. \\
$T_{N T C}$ & Temperature measured by the on-board \\
& NTC sensor at the DOC inlet \\
$T_{m}$ & Actual gas temperature at the DOC inlet. \\
$\dot{m}_{a}$ & Air mass flow measured by the on-board hot \\
$\dot{m}_{f}$ & Fuel mass flow estimated by the ECU. \\
$n$ & Engine speed. \\
$T_{m o d}$ & Mean value temperature model. \\
$T_{m o d}$ Virt & Virtual mean value modelled temperature. \\
$T_{T C, \bmod }$ & Modelled temperature. \\
$T_{N T C, m o d}$ Slow temperature filtered from $T_{T C}$. \\
$\hat{T}$ & Estimated temperature at the DOC inlet. \\
\hline
\end{tabular}

ple of $1.5 \mathrm{~mm}$ width, placed between the turbine and the engine block, was also used. Both thermocouples were connected to a PUMA measuring system with an acquisition frequency of $10 \mathrm{~Hz}$.

The NTC sensor is a Denso unit, able to measure in a range of temperatures from $-30^{\circ} \mathrm{C}$ to $1000^{\circ} \mathrm{C}$ with standard responsiveness, which error is $\pm 7^{\circ} \mathrm{C}$ at mid temperatures and $\pm 10^{\circ} \mathrm{C}$ at low and very high temperatures, according to the sensor manufacturer. Manufacturers of both sensors do not give information about response time. It was connected to a real time National Instruments PXI with an acquisition frequency of $1000 \mathrm{~Hz}$. Note that the NTC sensor was placed in the DOC canning itself, in front of the catalyst monolith, where it would be placed in on-board applications, whilst the fast thermocouple was connected in the exhaust line, as close as possible to the DOC-inlet.

An engine speed sensor and a hot film flow-meter were also used and connected to the engine ECU, which also provided the estimated injected fuel. The ECU signals had an acquisition frequency of $100 \mathrm{~Hz}$. A summary of the signals and variables used throughout the article can be found in Table 2 .

\section{System dynamics}

This section addresses the proper selection of the signals for the DOC-inlet temperature model. With this purpose, a study of the dynamics of the involved physical variables is carried out. Thus, the dynamics of the fuel path, the air path, the engine speed, and temperature sensors' measurements are evaluated. Tests shown in Figure 3 will be repeatedly used to identify the models coefficients along the paper. Tests consists of a tip in at $1500 \mathrm{rpm}$ (left plot) and a tip out at 2500 prm (right plot), where both air and fuel conditions vary.

\subsection{Fuel and air paths.}

Exhaust temperature is strongly dependent on the fuel injection and air mass flow. Fuel injection is controlled by the ECU, based on tabulated values, which mainly rely on rail pressure and injection duration. The error of these tables can be significant when short injections or split injection strategies are applied. Likewise, injector manufacturing discrepancies and ageing can create a significant unitto-unit (and cylinder-to-cylinder [21, 22]) dispersion in the actual injection quantity. Even so, ECU fuel mass estimation is fast and non-delayed, so it is able to perform cycleto-cycle variations [23]. Consequently, ECU injection signals have enough dynamic response to be used as input to the fast temperature model.

Air path of turbocharged engines has slower dynamics [24] due to mechanical, thermo- and fluid dynamic reasons. As stated by Alberer and del Re [17], Exhaust Gas Recirculation (EGR) dynamics are in between those of the fuel and the air path, so they may not be considered as an air path retarder. In any case, the air path dynamics are much faster than the heat transfer phenomena driving the exhaust gas temperature. Therefore, $\lambda^{-1}$ presents the fastest time response to an injection step, as shown in Figures 3 and $4 . \lambda^{-1}$ is calculated with the simple model of equation 1 and $S_{p}$ of Figure 4 represents the reached percentage of the step for each signal.

$$
\lambda^{-1}=\frac{\dot{m}_{a}}{14.5 \dot{m}_{f}}
$$

where $\dot{m}_{a}$ is the air mass flow measured by the hot film flowmeter, $\dot{m}_{f}$ is the fuel mass flow estimated by the ECU, and 14.5 stands for the diesel stoichiometric air-to-fuel ratio.

\subsection{Engine speed $n$.}

Engine speed has an important effect on exhaust gas temperature. Due to the measurement procedure itself, engine speed sensor dynamics allow to perform cylinder-tocylinder combustion diagnostics [25, 26], thus its dynamics response can be as well used as an input to the fast model.

\subsection{Pre-turbine temperature $T_{t u r b}$.}

Exhaust gas temperature dynamics are affected by those described before plus an added degree of freedom, which is the heat transmission between the gases generated in the combustion process and the engine block, valves and exhaust manifold. Its time response is slower than $\lambda^{-1}$, as can be seen in Figure 3 .

Figure 3 shows how engine cylinder-raw temperature $T_{\text {turb }}$ still increases after the initial transient phase of $\lambda^{-1}$, 

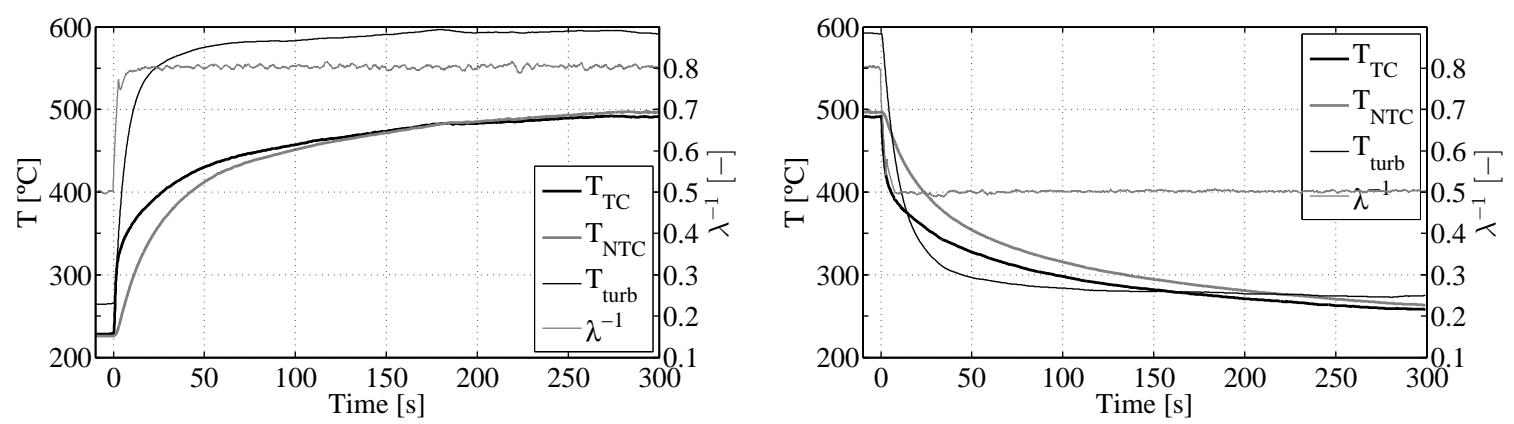

Fig. 3. Engine dynamics.
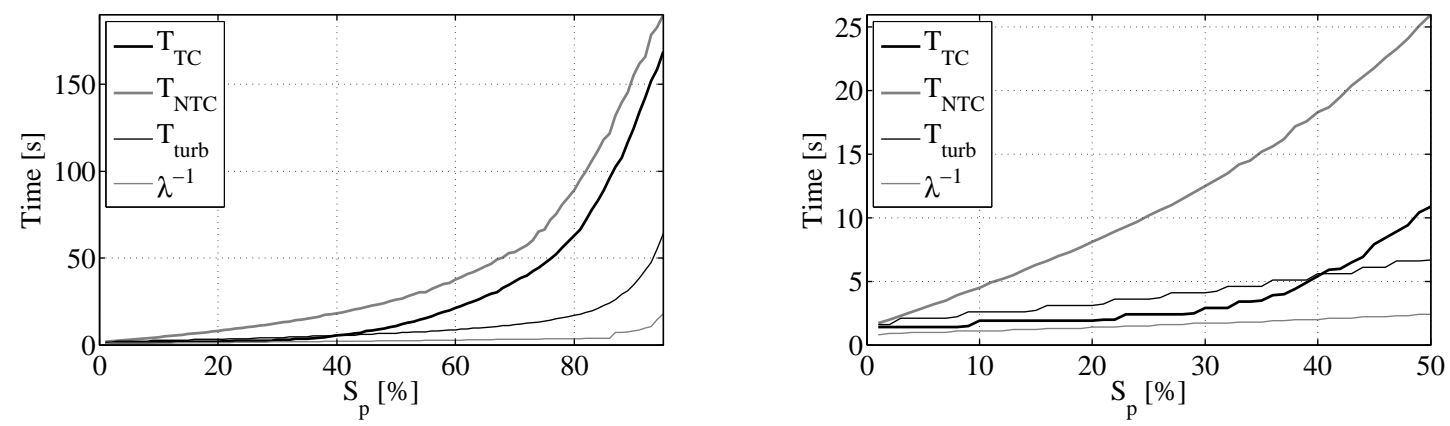

Fig. 4. Left: Time of the reached percentage of the signals at the tip in of Figure 3. Right: Left figure with zoom.

present during the first seconds after the load step. The initial steepest phase is due to in-cylinder changes, whilst the last phase with low slope is due to heat transmission effects of engine block and exhaust pipes. That is, cylinder gases temperature changes as fast as combustion settings change, but the surrounding metal follows slower heat transmission dynamics [27].

\subsection{DOC-inlet temperature}

The dynamics of the measurements at the DOC-inlet are analysed in this section for the two temperature sensors.

\subsubsection{Fast themocouple measurement $T_{T C}$.}

In this work, the thermocouple measurement $T_{T C}$, whose signal can be appreciated in Figure 3, is supposed to be the fastest possible measurement of the gas temperature. As depicted during the first $40 \%$ at the right side of Figure 4 , due to its lower thermal inertia, the fast thermocouple $T_{T C}$ observes the effect of the load step faster than $T_{T u r b}$. Afterwards, heat transmission effects accumulated by the exhaust manifold, the turbine and the exhaust line may be appreciated at Figure 3 as a low steep increase and at Figure 4 from $60 \%$ at the steepest stage. As $T_{T C}$ measurement is the best approximation to the real exhaust gas temperature, its signal will be considered hereinafter as the actual gas temperature.

\subsection{2. $N T C$ sensor measurement $T_{N T C}$}

The NTC sensor has the slowest dynamics response, as can be appreciated in Figures 3 and 4. The signal from the

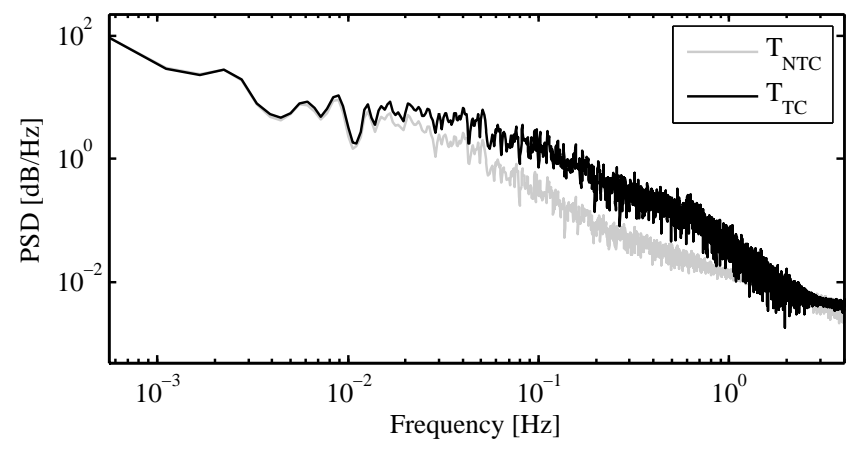

Fig. 5. $T_{T C}$ and $T_{N T C}$ presented in the frequency domain.

NTC sensor follows thermocouple measurements $T_{T C}$ when it comes to stationary engine operating conditions. Their time response is different due to its different masses, volumes, construction materials, locations and designs, which lead to different heat transmission due to conduction with the probe, convection with air, radiation with surrounding metals [14]. Despite these differences, temperature measurements have been traditionally modelled as first order systems, so that NTC sensor signal measurement will be considered as a first-order response of the TC measurement.

Figure 5 presents the measured $T_{T C}$ and $T_{N T C}$ in the frequency domain in order to show the effect of the sensor inertia on the temperature signals. For that purpose, a dynamic World harmonised Light vehicles Test Cycle (WLTC), which continuous transients cause continuous differences in sensors measurements, is used to generate the Power Spectral Density (PSD) of the signals. Frequency 
response of $T_{T C}$ and $T_{N T C}$ behave very similar at low frequencies, from $1 \mathrm{mHz}$ to $10 \mathrm{mHz}$. However, at the intermediate bandwidth there are noticeable differences, since the fast thermocouple presents more frequency content between $10 \mathrm{mHz}$ and $2 \mathrm{~Hz}$. At the highest frequencies shown in the plot, over $2 \mathrm{~Hz}$, the two signals behave similarly, which indicates that higher frequencies are not relevant.

\section{Temperature modelling}

In this part of the article, the different modelled systems are described. At first, the exhaust temperature model based on a look-up table, and afterwards, the rest of models describing the dynamics of the system. Procedures based on provoking step-like transitions can be used to identify and calibrate dynamic response of temperature sensors [28, 29]. However, due to heat transfer effects on the exhaust line, those step-like transitions applied in experimental tests are smoothed when exhaust mass flow arrives to TC and NTC sensors.

\subsection{DOC-inlet temperature model}

DOC-inlet temperature is modelled as a Mean Value Engine Model (MVEM) based on an engine map calibrated off-line, which depends on engine speed $n$ and fuel $m_{f}$ in $\mathrm{mg} / \mathrm{str}$. The engine map look-up table must be calculated off-line because learning algorithms for temperature are difficult to implement in real time. In this article, the lookup table employed has a density for $n$ of one point every $400 \mathrm{rpm}$ between 700 and $4500 \mathrm{rpm}$; and one point every $1 \mathrm{mg} / \mathrm{str}$ for $m_{f}$.

$$
T_{m o d, k}=\Theta\left(n_{k}, m_{f, k}\right)
$$

Engine mapping has been traditionally used in internal combustion engine modelling [30]. This option was preferred instead of an air path model, which is fast enough as stated in Section 3.1 although it would be more complex and possibly less robust. Other authors like Chen and Wang [31] or Eriksson [32] also propose control-oriented models based on look-up tables. Chen and Wang [31] use a turbine efficiency map whilst Eriksson [32] uses a temperature engine map. Although this model should be able to represent the temperature that the exhaust line temperature would reach after sufficient settling time, the actual purpose is to predict beforehand the temperature step, even with some uncertainty.

As depicted in Figure 6, the temperature increment provided by the model is representative of the temperature step. However, the steady state temperature does not perfectly match the final value, which can be seen in experimentation. To solve this issue, the bias will be modelled and the observer will handle the difference between measured and modelled values when it comes to steady state.

\section{2. $T_{T C}$ from $T_{\text {mod }}$}

Unfortunately, step-like transitions cannot be performed on-board for the characterisation of the sensors due to exhaust line heat transmission. Previous dynamics study in Section 3 has suggested two different dynamics in the exhaust line after a load step, one faster and one slower. This effect will be modelled as a second order system, in order to obtain the signal of $T_{T C}$ from $T_{\text {mod }}$. One pole (eq. 3 ) is meant to represent fast dynamics due to in-cylinder changes, whilst the other pole (eq. 4) is meant to represent slow dynamics due to heat transmission effects in the exhaust line. Model equations are:

$$
\begin{gathered}
T_{1}=\frac{(1-b) d}{1-b z^{-1}} T_{\text {mod }} z^{-\tau / T_{s}} \\
T_{2}=\frac{(1-c)(1-d)}{1-c z^{-1}} T_{\text {mod }} z^{-\tau / T_{s}} \\
T_{T C, \text { mod }}=T_{1}+T_{2}
\end{gathered}
$$

where $\mathrm{z}$ is the $\mathrm{z}$-transform variable, $b$ is the filtering quantity of the fast pole, $c$ is the filtering quantity of the slow pole, $d$ is a factor to weight both fast and slow poles, $\tau$ is the model-to-sensor pure delay and $T_{s}$ is the sampling period.

A dynamic characterisation of the sensor is mandatory to identify eq. (5). As seen in the previous Figure 6, model accuracy may not be perfect, so a synthetic signal of the ideal $T_{\text {mod }}$ value has been generated to calibrate the second order system. Therefore, the second order discrete filter is applied to a virtual signal of $T_{\text {mod }}$, named $T_{\text {mod,virt }}$, as represented in Figure 7 . Note that this synthetic signal can only be generated off-line, since it is calculated from the steady-state value of $T_{T C}$.

Even if the measurements with the sensor are calibrated, it remains an inherent error because time invariant models for sensors are not completely realistic. Although modelling shows good results, model parameters may depend on operating conditions, manufacturing discrepancies and others, which may lead to uncontrolled behaviours.

Results of the system identification can be seen in Figure 7. Identified values for the coefficients are: $b=0.94, c=0.998$ and $d=0.5$. These coefficients are kept constant for all engine operating conditions and are calculated off-line. The delay, $\tau$, between $T_{m o d}$ and the sensors' measurements is not higher than 1 second, as can be seen in Figures 4 and 6 . Therefore, its value can be considered negligible in comparison with the dynamics, of dozens of seconds, of the system.

Even if dynamics could be appreciated in time domain, the bias would make difficult to validate the modelled temperature by comparing it with the actual measured temperature. Therefore, performance of the model is discussed in the frequency domain in Figure 8. A WLTC test is used for this validation because of its highly different conditions of exhaust mass flows and temperatures. Plots are slightly filtered in order to reduce possible confusion due to the 

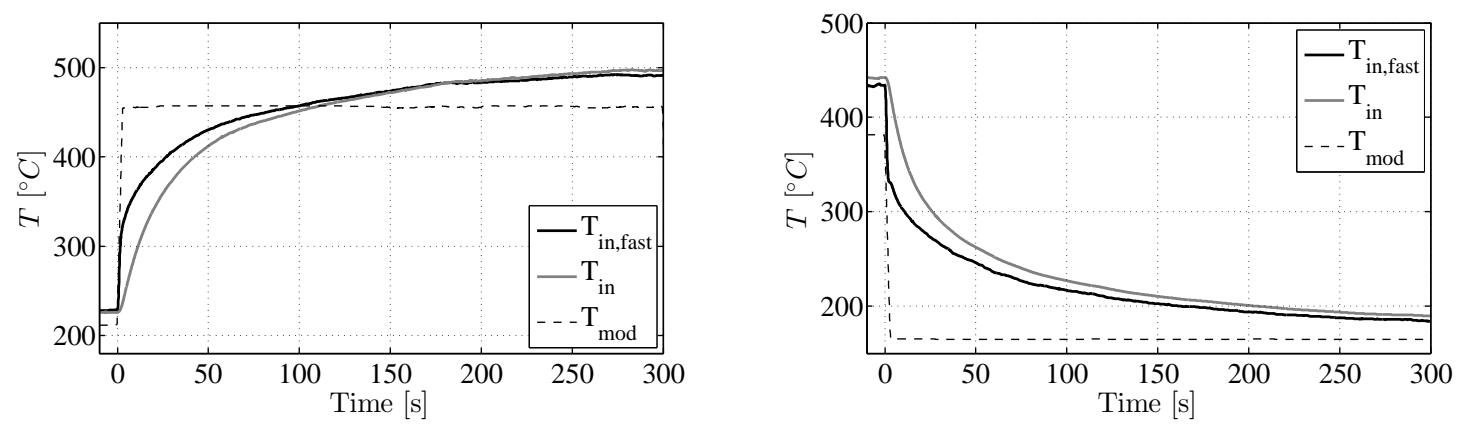

Fig. 6. Mean value temperature model applied to a load step.
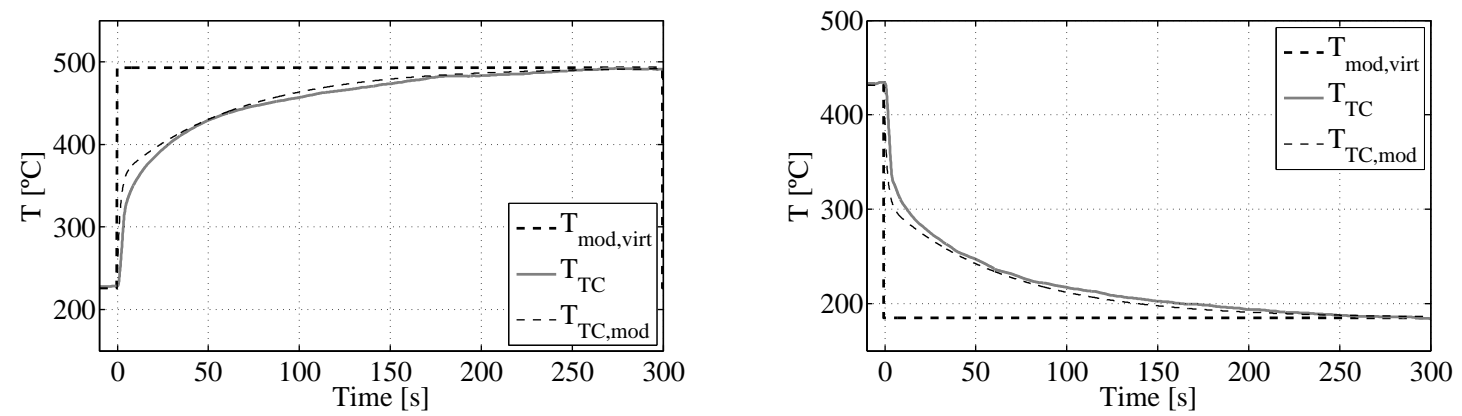

Fig. 7. Identification result of the second order system applied to $T_{\text {mod }}$ to obtain $T_{T C, \bmod }$.

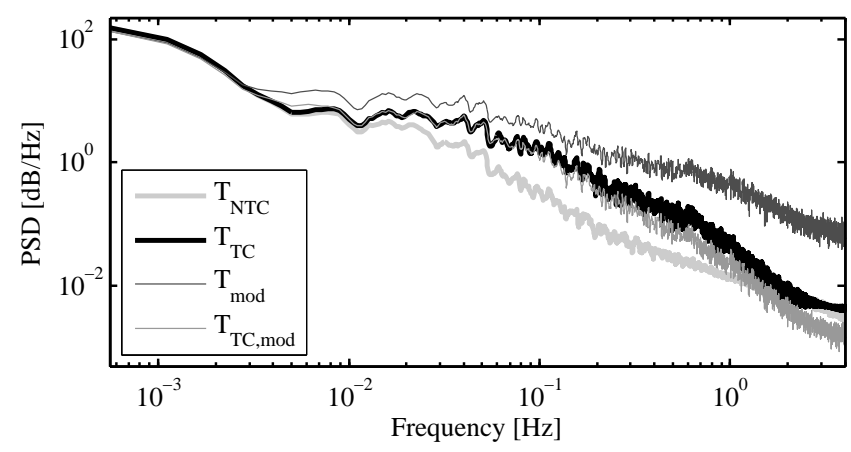

Fig. 8. Validation of the $T_{\bmod }$ to $T_{T C}$ model in the frequency domain.

number of different variables represented and their original high noise level.

It can be appreciated how the second order filter reduces the frequency content of $T_{\text {mod }}$ until its signal matches $T_{T C}$. $T_{T C \text {,mod }}$ temperature presents slightly less frequency content than $T_{T C}$ at high frequencies, although it is still higher than $T_{N T C}$. At the highest frequencies present in the plot, the frequency content of $T_{T C, \text { mod }}$ is even lower than that of $T_{N T C}$, although in this range, frequency content of $T_{N T C}$ is almost equal to $T_{T C}$, which may be associated to noise.

Model output $T_{T C, \bmod }$ can be related to the actual value of $T_{T C}$ considering a bias $\theta$ :

$$
T_{T C}=T_{T C, \bmod }+\theta
$$

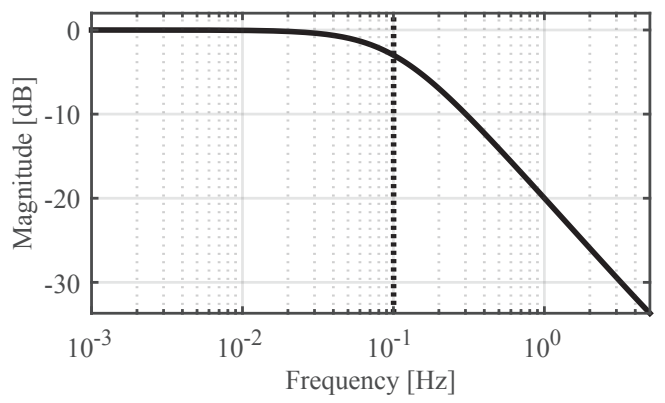

Fig. 10. Magnitude Bode of the first order system applied to $T_{T C}$ to obtain $T_{N T C, \bmod }$.

\section{3. $T_{N T C}$ from $T_{T C}$}

Although other model structures could be used, a discrete linear first order model is chosen to represent the temperature measurement of the NTC sensor with respect to the thermocouple. Modelling equation results in:

$$
T_{N T C, \bmod }=T_{N T C}+\nu=\frac{1-a}{1-a z^{-1}} T_{T C}+\nu
$$

where $a$ is the filtering quantity and the main parameter, since no delay between sensors' measurements is considered due to its closed location. A noise $\nu$ has been added to the delayed and filtered value $T_{T C}$ in order to represent the measurement noise and other uncontrolled effects. Results of the system identification can be seen in Figure 9 .

The bode plot of the identified system, if $\mathrm{a}=0.986$, is shown in Figure 10, which has been calculated by transforming the transfer function in the $\mathrm{z}$ domain to the $\mathrm{s}$ do- 

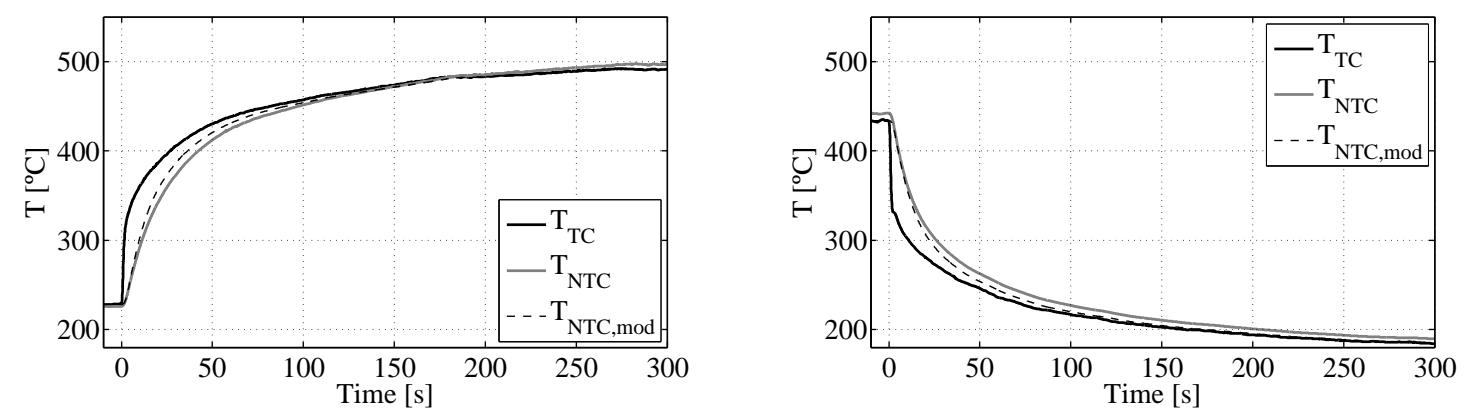

Fig. 9. Identification result of the first order system applied to $T_{T C}$ to obtain $T_{N T C, \bmod }$.

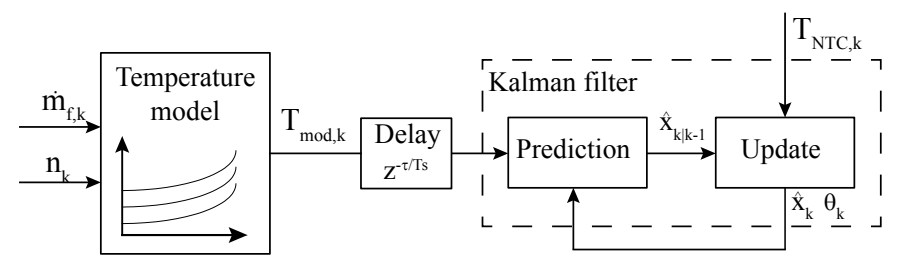

Fig. 11. Scheme of the proposed estimation procedure.

main through the Tustin method [33, 34]. The cut-off frequency, established when bode gain is $-3 \mathrm{~dB}$, is $0.14 \mathrm{~Hz}$. Although the obtained value of the coefficient $a$ has been used throughout the paper, some effect of operating conditions on the optimal filtering quantity $a$ is expected.

\section{Fast temperature estimation}

A Kalman filter is used in order to fuse data from the sensor measurement and the model. Kalman filter behaves as a low pass filter for the temperature model and as a high pass filter for the sensor measurement [35]. Through the combination of the sensor measurement and the model information, the Kalman filter is used to track the value of the bias $\theta$ between $T_{T C}$ and $T_{T C \text {,mod }}$, thus providing an estimate of $\hat{T}_{T C}$.

Figure 11 shows a scheme of the proposed controller structure. All calculations and equations are set-up in discrete form with a constant sampling frequency $f_{s}$ of $10 \mathrm{~Hz}$. Main inputs are injected fuel mass $m_{f}$, engine speed $n$ and NTC sensor measurement $T_{N T C}$.

\subsection{Observer design}

The proper combination of equations (2)-(7) allows to write the system in the following linear discrete-time statespace representation:

$$
\begin{gathered}
\mathbf{x}_{\mathbf{k}}=\mathbf{A x}_{\mathbf{k}-\mathbf{1}}+\mathbf{B} u_{k}+\mathbf{W}_{\mathbf{k}} \\
y_{k}=\mathbf{C x}_{\mathbf{k}}+\nu_{k}
\end{gathered}
$$

where $\mathbf{x}_{\mathbf{k}}$, the states vector of the system; $y_{k}$, the NTC temperature measurement; and $u_{k}$, the model input, are defined as:

$$
\begin{gathered}
\mathbf{x}_{\mathbf{k}}=\left[\begin{array}{lllll}
\theta & T_{1} & T_{2} & T_{T C, \bmod } & T_{N T C, \bmod }
\end{array}\right]_{\mathbf{k}}^{T} \\
y_{k}=T_{N T C, k} \\
u_{k}=T_{m o d, k-\tau / T_{s}}
\end{gathered}
$$

The description of the system matrices (8) is:

$$
\mathbf{A}=\left[\begin{array}{ccccc}
1 & 0 & 0 & 0 & 0 \\
0 & b & 0 & 0 & 0 \\
0 & 0 & c & 0 & 0 \\
1 & 1 & 1 & 0 & 0 \\
0 & 0 & 0 & 1-a & a
\end{array}\right]
$$

$$
\begin{aligned}
& \mathbf{B}=\left[\begin{array}{lllll}
0 & (1-b) d & (1-c)(1-d) & 0 & 0
\end{array}\right]^{T} \\
& \mathbf{C}=\left[\begin{array}{lllll}
0 & 0 & 0 & 0 & 1
\end{array}\right] \\
& \mathbf{W}_{\mathbf{k}}=\left[\begin{array}{lllll}
w_{k} & 0 & 0 & 0 & 0
\end{array}\right]^{T}
\end{aligned}
$$

The selected distribution of noises considers that a white noise is related to the sensor measurement $\left(v_{k}\right)$ and other white noise is related to the model $\left(w_{k}\right)$. Kalman [36] addressed the optimal solution of the estimation problem, i.e. estimating the state $x$ of the system from the evolution of $y$ and $u$. In a first step, the state is estimated considering the system inputs and their expected dynamic characteristics:

$$
\hat{\mathbf{x}}_{\mathbf{k}-\mathbf{k - 1}}=\mathbf{A} \hat{\mathbf{x}}_{\mathbf{k}-\mathbf{1}}+\mathbf{B} u_{k}
$$

and in a second step the a priori estimate of the state $\hat{\mathbf{x}}_{\mathbf{k}-\mathbf{k - 1}}$ is updated using the error calculation $e_{k}$ and the Kalman filter gain $\mathbf{K}_{\mathbf{k}}$ :

$$
\begin{gathered}
e_{k}=y_{k}-\mathbf{C} \hat{\mathbf{x}}_{\mathbf{k}-\mathbf{k}-\mathbf{1}} \\
\hat{\mathbf{x}}_{\mathbf{k}}=\hat{\mathbf{x}}_{\mathbf{k}-\mathbf{k}-\mathbf{1}}+\mathbf{K}_{\mathbf{k}} e_{k}
\end{gathered}
$$

where the Kalman gain $\mathbf{K}_{\mathbf{k}}$ could be generally obtained online with the covariance matrices of the noises distribution by solving the Riccati equation. However, the considered system is Linear Time Invariant (LTI) and fully observable system, then the Kalman gain is constant. Then, it can be 
Table 3

Values of the parametric study.

\begin{tabular}{lllll}
\hline Coefficient & Value & \multicolumn{2}{l}{ RMSE $R^{2}$} & IE \\
\hline$a_{1}$ & 0.9 & 217.7 & 0.14 & $-39,199$ \\
$a_{2}$ & 0.986 & 11.7 & 0.998 & 6,760 \\
$a_{3}$ & 0.999 & 29.2 & 0.985 & 11,392 \\
$\sigma_{w}^{2} / \sigma_{v, 1}^{2}$ & 0.01 & 13.1 & 0.997 & 9,054 \\
$\sigma_{w}^{2} / \sigma_{v, 2}^{2}$ & 50 & 11.7 & 0.998 & 6,760 \\
$\sigma_{w}^{2} / \sigma_{v, 3}^{2}$ & 5000 & 13.6 & 0.997 & 6,579 \\
\hline
\end{tabular}

calculated off-line with the variances of the noises $\sigma_{w}^{2}$ and $\sigma_{u}^{2}$ by an iterative process:

$$
\mathbf{K}_{\infty}\left(\sigma_{w}^{2} / \sigma_{v}^{2}\right)=\lim _{k \rightarrow+\infty} \mathbf{K}_{\mathbf{k}}
$$

The use of a constant gain $\mathbf{K}_{\infty}$ avoids $\mathbf{K}_{\mathbf{k}}$ calculation in each iteration using the Riccati equation, so it is more efficient in terms of ECU computational resources. Eventually, temperature estimation is the fourth state of the state-space vector:

$$
\hat{T}=\hat{T}_{T C, \bmod }
$$

\subsection{Algorithm tuning}

Algorithm tuning consists in the calibration of the system coefficients $a, b, c, d$ and $\tau$, which have been previously obtained in the paper for each system independently, as well as the calibration of the observer.

Observer tuning consists in the calibration of sensor $v_{k}$ and model $w_{k}$ noises, which are considered as Gaussian distributions with zero mean and variances given by $\sigma_{w}^{2}$ and $\sigma_{v}^{2}$. Uncertainties in sensor characterisation and bias cancelling performance make difficult the selection of an optimal noises combination. According to Payri et al. [37], the time needed to cancel the bias depends on the variances fraction $\sigma_{w}^{2} / \sigma_{v}^{2}$.

An analysis of $a, b, c, d, \tau$ and $\sigma_{w}^{2} / \sigma_{v}^{2}$ reveals that $a$ and $\sigma_{w}^{2} / \sigma_{v}^{2}$ have major impact on the calibration. Therefore, a parametric study of how these two variables affect estimation dynamics is shown in Figure 12 for 3 different values of each coefficient. Table 3 contains those values with their corresponding errors: the root mean squared error (RMSE), the squared error $\left(R^{2}\right)$, or determination coefficient, and the integral error (IE). The second value corresponds to the final calibration, whilst the first value is lower and the third is higher. The rest of coefficients $b, c$ and $d$ are those used for the fast temperature estimation.

In both plots of Figure 12, the estimated temperature is shown as the intermediate line of the parametric study. The calibrated value for $a$, found with the step test, results in a good characterisation of the sensor dynamics, whilst $\sigma_{w}^{2} / \sigma_{v}^{2}$ has been calibrated using the PSD. Top plot shows how by varying $a$, the estimation has more ( $a$ decreases) or less ( $a$ increases) frequency content. Bottom plot shows how by varying $\sigma_{w}^{2} / \sigma_{v}^{2}$, frequency content at high frequencies is increased $\left(\sigma_{w}^{2} / \sigma_{v}^{2}\right.$ increases $)$ or decreased $\left(\sigma_{w}^{2} / \sigma_{v}^{2}\right.$ decreases $)$. $\sigma_{w}^{2} / \sigma_{v}^{2}$ also marks from which frequency the variation has impact on the PSD. The errors study shows the best results for the calibrated values.

The parametric study of $a$ and $\sigma_{w}^{2} / \sigma_{v}^{2}$ in Figure 12 points out how the observer could increase its frequency content if necessary by decreasing $a$ and increasing $\sigma_{w}^{2} / \sigma_{v}^{2}$. In this sense, the second order system applied to $T_{\text {mod }}$ could apply a less severe filter by modifying its coefficients. That is, adapting the presented algorithm to other pair of sensors can be done by re-tuning the coefficients. Then, in case the thermocouple used were not be able to reproduce the actual temperature due to thermal inertia and it were necessary, response speed of the estimation could be increased by coefficients tuning.

\section{Results}

Experimental validation of the algorithm is carried out in this section. Validation tests use the fast thermocouple measurement as a reference to compare with the estimated temperature.

Results of the calibration for the WLTC, in time domain during a temporal window, can be seen in Figure 13. Despite minor differences in severe peaks and valleys, estimation matches quite well the temperature measured by the thermocouple. The analysis made in the frequency domain for this test may explain the high performance achieved for the temperature estimation in this test.

A test that stresses the dynamics of the system, with high steps of exhaust mass flow and temperature, is shown in Figure 14. A difference in steady-state temperature can be noticed between NTC and TC measurements, due to its slight different location in the exhaust line: NTC sensor is slightly closer to the DOC ceramics, which may interfere in sensor measurements. In some cases, and due to nonmodelled phenomena with a first order sensor model, there is an overshoot of estimated temperature at the steps. Even so, it can be appreciated that during a transient, the estimated temperature $\hat{T}$ performs faster than the NTC measurement, matching the thermocouple signal $T_{T C}$.

Algorithm performance is eventually proved in a NEDC for a time window of the cycle. Results of the NEDC (Figure 15) show that even if model has some difference with the measured TC temperature due to the effect of operating conditions, it is able to reproduce fast transients. Differences are mainly appreciated during valleys of temperature, where uncertainties in modelling may cause underestimations of measured $T_{T C}$ temperature. During nontransient phases, estimated value is the $T_{N T C}$ signal.

\section{Conclusions}

The transient response of the robust DOC-inlet NTC temperature sensor has been improved by means of a signal 

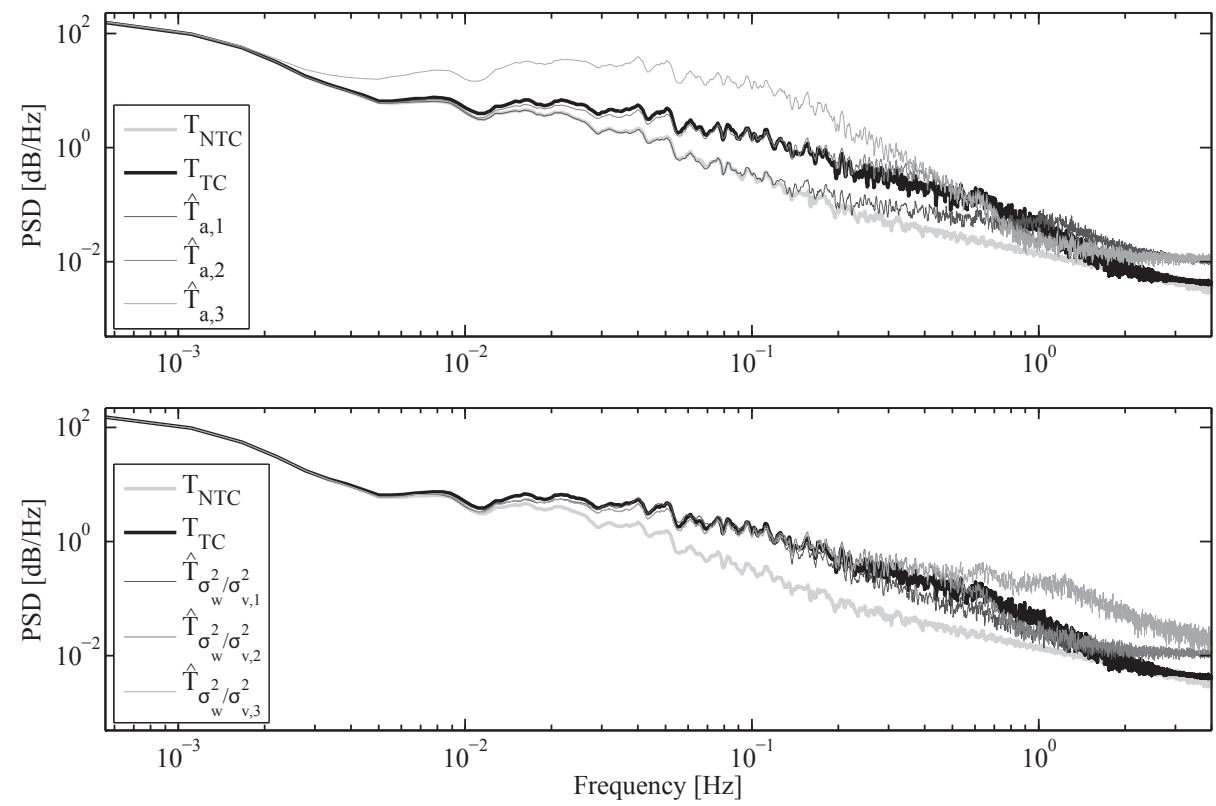

Fig. 12. Sensitivity analysis of $a$ (Top plot) and $\sigma_{w}^{2} / \sigma_{v}^{2}$ (Bottom plot) in the frequency domain.

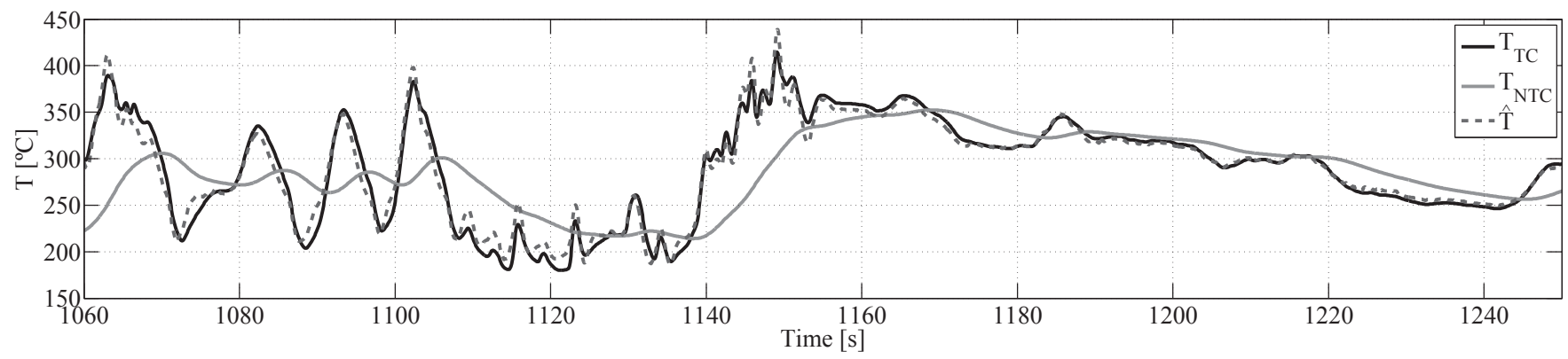

Fig. 13. Result of estimated temperature in a time window of a WLTC.

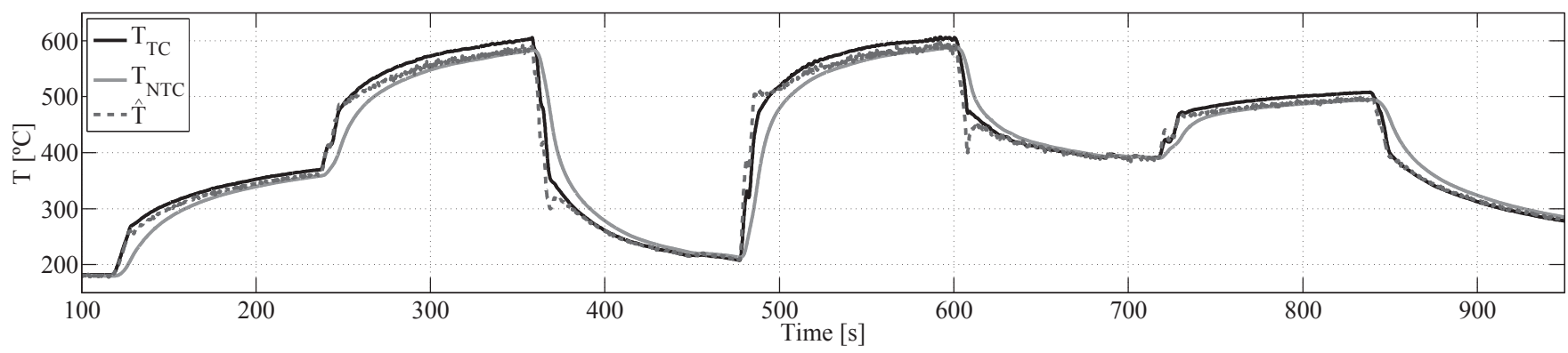

Fig. 14. Result of estimated temperature in a time window of a test with high steps in engine operating conditions.

reconstruction technique, which algorithm is to be used in production vehicles. With this approach, an on-board DOC model could be sensitively improved due to several reasons. For instance, it would measure an oxidation phase [6] when $\hat{T}_{T C}>L O T$, which is usually about $200{ }^{\circ} \mathrm{C}$; and it could better reproduce dynamics at the DOC-outlet. Therefore, the estimated temperature can be used to improve the aftertreatment systems efficiency and its diagnostics.

Regarding the algorithm, a first order model has been used to model the transient response of the NTC temperature sensor with respect to the thermocouple measurement.
Through a dynamics study, engine speed and fuel injection have been the selected inputs to the fast model, which is based on an engine look-up table and a second order filter system. One fast pole represents the fast in-cylinder dynamics, whilst the second and slower pole represents heat transmission through the exhaust line. The presented methodology combines data from a fast but biased temperature model with a slow but accurate NTC sensor. A constant Kalman filter gain, calculated off-line, is used to estimate the states of the linear time invariant state-space model.

Algorithm tuning has been divided into several steps, in 


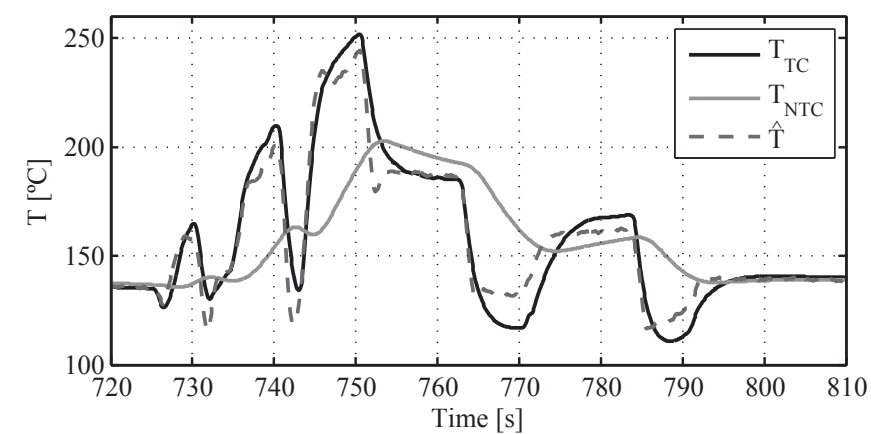

Fig. 15. Result of estimated temperature in a time window of a NEDC.

some of which a frequency analysis has been performed. The signal reconstruction has been successfully achieved, as results show in European approval test cycles and dynamic tests. Validation results, made in tests representative of real driving conditions, demonstrate its feasibility for being used in commercial vehicles.

\section{Acknowledgements}

This research has been partially financed by the Spanish Ministerio de Economia y Competitividad, through project TRA2013-40853-R PRELIMIT.

\section{References}

[1] R. Sutjiono, P. Tayal, K. Zhou, P. Meckl, Real-Time On-Board Indirect Light-Off Temperature Estimation as a Detection Technique of Diesel Oxidation Catalyst Effectiveness Level, in: SAE Technical Paper, SAE International, 2013.

[2] I. Nova, E. Tronconi, Urea-SCR technology for deNOx after treatment of Diesel exhausts, Springer, 2014.

[3] Y.-W. Kim, M. V. Nieuwstadt, G. Stewart, J. Pekar, Model Predictive Control of DOC Temperature during DPF Regeneration, in: SAE Technical Paper, SAE International, 2014.

[4] A. Frobert, Y. Creff, O. Lepreux, L. Schmidt, S. Raux, Generating Thermal Conditions to Regenerate a DPF: Impact of the Reductant on the Performances of Diesel Oxidation Catalysts, in: SAE Technical Paper, SAE International, 2009.

[5] T. V. Johnson, Review of Diesel Emissions and Control, SAE Int. J. Fuels Lubr. (2010) 16-29.

[6] C. Guardiola, B. Pla, J. Mora, D. Lefebvre, Control Oriented Model for Diesel Oxidation Catalyst Diagnosis, IFAC-PapersOnLine 48 (15) (2015) 427 - 433.

[7] M. V. Nieuwstadt, D. Upadhyay, F. Yuan, Diagnostics for Diesel Oxidation Catalysts, in: SAE Technical Paper, SAE International, 2005.

[8] J. Riegel, H. Neumann, H.-M. Wiedenmann, Exhaust gas sensors for automotive emission control, Solid State Ionics 152 (2002) 783-800.
[9] C. regulation No 719/2007, Regulation (EC) No $715 / 2007$ of the European Parliament and of the Council of 20 June 2007 on type approval of motor vehicles with respect to emissions from light passenger and commercial vehicles (Euro 5 and Euro 6) and on access to vehicle repair and maintenance information .

[10] C. regulation No 459/2012, Amending Regulation EC No $715 / 2007$ of the European Parliament and of the Council and Commission Regulation (EC) No $692 / 2008$ as regards emissions from light passenger and commercial vehicles (Euro 6) .

[11] T. Johnson, Vehicular Emissions in Review, SAE Int. J. Engines 7 (2014) 1207-1227.

[12] N. El-Awar, D. Geer, T. Krellner, P. Straub, Automotive temperature sensing, Keystone Thermometrics Corporation Application Notes .

[13] K. Mollenhauer, H. Tschöke, Handbook of diesel engines, Springer-Verlag Berlin Heidelberg 2010, 2010.

[14] K. Kar, S. Roberts, R. Stone, M. Oldfield, B. French, Instantaneous exhaust temperature measurements using thermocouple compensation techniques, SAE SP (2004) 169-190.

[15] R. J. Kee, P. Hung, B. Fleck, G. Irwin, R. Kenny, J. Gaynor, S. McLoone, Fast Response Exhaust Gas Temperature Measurement in IC Engines, in: SAE Technical Paper, SAE International, 2006.

[16] R. Schultz, P. H. Meckl, Light-Off Temperature Shift for Catalyzed Diesel Particulate Filter On-Board Diagnostics, in: SAE Technical Paper, SAE International, 2012.

[17] D. Alberer, L. del Re, Optimization of the transient Diesel engine operation, in: SAE Technical Paper, Consiglio Nazionale delle Ricerche, 2009.

[18] L. Del Re, F. Allgöwer, L. Glielmo, C. Guardiola, I. Kolmanovsky, Automotive model predictive control: models, methods and applications, vol. 402, Springer, 2010.

[19] D. Alberer, Fast Oxygen Based Transient Diesel Engine Control, Advances in mechatronics, Trauner, 2012.

[20] C. Guardiola, H. Climent, B. Pla, D. BlancoRodriguez, ECU-oriented models for NOx prediction. Part 2: adaptive estimation by using an NOx sensor, Proceedings of the Institution of Mechanical Engineers, Part D: Journal of Automobile Engineering 229 (10) (2015) 1345-1360.

[21] V. Macian, J. M. Luján, C. Guardiola, P. Yuste, DFTbased controller for fuel injection unevenness correction in turbocharged diesel engines, Control Systems Technology, IEEE Transactions on 14 (5) (2006) 819827.

[22] V. Macián, J. Luján, C. Guardiola, A. Perles, A comparison of different methods for fuel delivery unevenness detection in Diesel engines, Mechanical systems and signal processing 20 (8) (2006) 2219-2231.

[23] F. Payri, J. Luján, C. Guardiola, G. Rizzoni, Injection diagnosis through common-rail pressure measure- 
ment, Proceedings of the Institution of Mechanical Engineers, Part D: Journal of Automobile Engineering 220 (3) (2006) 347-357.

[24] J. Galindo, J. Luján, H. Climent, C. Guardiola, Turbocharging system design of a sequentially turbocharged diesel engine by means of a wave action model, Tech. Rep., SAE Technical Paper, 2007.

[25] F. T. Connolly, G. Rizzoni, Real time estimation of engine torque for the detection of engine misfires, Journal of dynamic systems, measurement, and control 116 (4) (1994) 675-686.

[26] D. Lee, G. Rizzoni, Detection of partial misfire in IC engines using a measurement of crankshaft angular velocity, Tech. Rep., SAE Technical Paper, 1995.

[27] M. Mrosek, H. Sequenz, R. Isermann, Identification of emission measurement dynamics for diesel engines, IFAC proceedings volumes (IFAC-PapersOnline) 18 (2011) 11839-11844.

[28] K. Ogata, Modern control engineering, Prentice Hall PTR, 2009.

[29] J. Galindo, J. Serrano, C. Guardiola, D. BlancoRodriguez, I. Cuadrado, An on-engine method for dynamic characterisation of NO $\mathrm{x}$ concentration sensors, Experimental Thermal and Fluid Science 35 (3) (2011) 470-476.

[30] L. Guzzella, C. Onder, Introduction to modeling and control of internal combustion engine systems, Springer Science \& Business Media, 2009.

[31] P. Chen, J. Wang, Control-oriented model for integrated diesel engine and aftertreatment systems thermal management, Control Engineering Practice 22 (2014) 81-93.

[32] L. Eriksson, Mean Value Models for Exhaust System Temperatures, in: SAE Technical Paper, SAE International, 2002.

[33] G. Proakis, G. Manolakis, Digital signal processing: principles, algorithms, and applications, Pentice Hall .

[34] F. Haugen, Discrete-time signals and systems, Tech, 2005.

[35] C. Guardiola, B. Pla, D. Blanco-Rodriguez, A. Mazer, O. Hayat, A bias correction method for fast fuel-to-air ratio estimation in diesel engines, Proceedings of the Institution of Mechanical Engineers, Part D: Journal of Automobile Engineering 227 (8) (2013) 1099-1111.

[36] R. E. Kalman, A new approach to linear filtering and prediction problems, Journal of basic Engineering 82 (1) (1960) 35-45.

[37] F. Payri, C. Guardiola, D. Blanco-Rodriguez, A. Mazer, A. Cornette, Methodology for Design and Calibration of a Drift Compensation Method for FuelTo-Air Ratio Estimation, in: SAE Technical Paper, SAE International, 2012. 